\title{
Empirical estimates of the Galactic halo contribution to the dispersion measures of extragalactic fast radio bursts using X-ray absorption
}

\author{
Sanskriti Das, ${ }^{1 \star}$ Smita Mathur, ${ }^{1,2}$ Anjali Gupta, ${ }^{1,3}$ Fabrizio Nicastro ${ }^{4,5}$ and Yair Krongold ${ }^{6}$ \\ ${ }^{1}$ Department of Astronomy, The Ohio State University, 140 West 18th Avenue, Columbus, OH 43210, USA \\ ${ }^{2}$ Center for Cosmology and Astroparticle Physics, 191 West Woodruff Avenue, Columbus, OH 43210, USA \\ ${ }^{3}$ Columbus State Community College, 550 E Spring St., Columbus, OH 43210, USA \\ ${ }^{4}$ Observatorio Astronomico di Roma - INAF, Via di Frascati 33, 1-00040 Monte Porzio Catone, RM, Italy \\ ${ }^{5}$ Harvard-Smithsonian Center for Astrophysics, 60 Garden St., MS-04, Cambridge, MA 02138, USA \\ ${ }^{6}$ Instituto de Astronomia, Universidad Nacional Autonoma de Mexico, 04510 Mexico City, Mexico
}

22 October 2020

\begin{abstract}
We provide an empirical list of the Galactic dispersion measure $\left(D M_{G a l}\right)$ contribution to the extragalactic fast radio bursts along 72 sightlines. It is independent of any model of the Galaxy, i.e., we do not assume the density of the disk or the halo, spatial extent of the halo, baryonic mass content, or any such external constraints to measure $D M_{G a l}$. We use $21-\mathrm{cm}, \mathrm{UV}$, EUV and $\mathrm{X}$-ray data to account for different phases, and find that $D M_{G a l}$ is dominated by the hot phase probed by X-ray absorption. We improve upon the measurements of $\mathrm{N}\left(\mathrm{O}_{\mathrm{VII}}\right)$ and $\mathrm{f}_{O V I I}$ compared to previous studies, thus providing a better estimate of the hot phase contribution. The median $D M_{G a l}=64_{-23}^{+20} \mathrm{~cm}^{-3}$ pc, with a $68 \%(90 \%)$ confidence interval of 33-172 (23-660) $\mathrm{cm}^{-3}$ pc. The $D M_{G a l}$ does not appear to follow any trend with the galactic longitude or latitude, and there is a large scatter around the values predicted by simple disk+spherical halo models. Our measurements provide more complete and accurate estimates of $D M_{G a l}$ independent from the previous studies. We provide a table and a code to retrieve $D M_{G a l}$ for any FRB localized in the sky.
\end{abstract}

Key words: Galaxy: halo-X-rays: diffuse background-radio continuum: transients-(galaxies:) quasars: absorption lines(galaxies:) intergalactic medium

\section{INTRODUCTION}

Fast radio bursts (FRBs) are bright ( $50 \mathrm{mJy}-100 \mathrm{Jy}$ ) coherent pulses of emission at radio frequencies, with duration of order milliseconds or less (Lorimer et al. 2007; Petroff et al. 2019). The intervening plasma through which the pulses travel imposes a refractive index that retards the group velocity as a function of frequency. This leads to a time delay $(\Delta t)$ between the highest $\left(v_{h}\right)$ and lowest $\left(v_{l}\right)$ radio frequencies of the pulse, quantified by the dispersion measure (DM): $D M \propto \Delta t /\left(v_{l}^{-2}-v_{h}^{-2}\right)$. The DM of an FRB at redshift $z$ is defined as $D M=\int \frac{n_{e}}{1+z} d l$, a line-of-sight integration of the free-electron number density of the intervening medium. Typically, the DM of FRBs are hundreds (and sometimes a few thousands) of $\mathrm{cm}^{-3} \mathrm{pc}$ (Petroff et al. 2016), which is too large to be explained by the free electrons in the interstellar medium (ISM) of the Milky Way (Cordes \& Lazio 2002; Dolag et al. 2015; Yao et al. 2017). This indicates that FRBs are extragalactic.

The extragalactic origin of FRBs makes it a promising tool to probe the otherwise invisible ionized intervening medium. Over the past decade, many uses of the DM of FRBs have been proposed, such as to study the cosmic reionization history, large-scale structure of the universe, cosmic proper distance measurements, baryon fraction of the intergalactic medium (IGM), and precision cosmology (Zheng

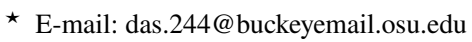

et al. 2014; Masui \& Sigurdson 2015; Yu \& Wang 2017; Li et al. 2019; Macquart et al. 2020, and references therein).

The observed DM toward FRBs includes the DM of the host galaxy $\left(D M_{\text {host }}\right)$, the intergalactic medium (IGM; $\left.D M_{I G M}\right)$, the Local Group, and the Milky Way. For any cosmological calculation using the DM of FRBs, it is necessary to know and remove the Galactic contribution, $D M_{G a l}$ from the total observed DM. By Galactic, we mean primarily the ISM in the disk and the circumgalactic medium (CGM) in the halo of the Milky Way. Because of the unknown spatial extent of the Galactic halo, the DM signatures of the Local Group and the Galaxy halo become observationally indistinguishable, broadly providing the $z \approx 0$ value.

The surveys searching for FRBs usually set a cutoff on the DM such that the DM of a detected FRB is larger than $D M_{G a l}$ (Petroff et al. 2019). The $\mathrm{DM}_{I G M}-z$ relation can be used to roughly estimate the redshift of an FRB (Zheng et al. 2014; Li et al. 2019), but to estimate $D M_{I G M}$ the knowledge of $D M_{G a l}$ is essential. Therefore, it is important to have a detailed understanding of the sky distribution of the Galactic DM to efficiently detect FRBs and to measure their distances, which again is instrumental for cosmological studies.

Because of the larger sky coverage than the Galactic sightlines, extragalactic sightlines are crucial for studying the IGM. Therefore, it is necessary to accurately estimate the contribution of Galactic halo along the extragalactic sightlines. Yamasaki \& Totani (2020) prescribed a disk-like plus a spherical halo density model from the X- 
ray emission measure of the Galactic halo along > 100 sightlines (see also Keating \& Pen 2020) and predicted the Galactic DM contribution based on that model. This model is complementary to the previous models (e.g., Cordes \& Lazio 2002; Yao et al. 2017) around the disk where the hot gas and other cooler phases have comparable contribution, but at high galactic latitude $\left(|b| \geqslant 20^{\circ}\right)$ this model is a significant improvement over previous models which ignored the halo component for simplicity.

As emission measure (EM) is proportional to the density squared $\left(\mathrm{EM}=\int n_{e} n_{p} d l\right)$, it is not possible to retrieve the dispersion measure from the EM without constructing a density model. Often, such models depend on many parameters including the spatial extent of the Galactic halo, the baryon fraction in the halo and the virial mass of Milky Way. However, none of these quantities are well-constrained and the spatial extent varies wildly all over the sky (see BoylanKolchin et al. 2013; Gupta et al. 2012, 2017, for details). This leads to a huge systematic uncertainty which usually surpasses the statistical uncertainty of the emission measurements from which the density model is constructed.

On the other hand, the column density $\left(N_{X}=\int n_{X} d l\right)$ from absorption analyses can be directly converted to the dispersion measure assuming some ionization condition. Prochaska \& Zheng (2019) used the column densities of O vir K- $\alpha$ lines from Fang et al. (2015) to calculate the DM contribution of the hot Galactic halo and constrained their proposed halo model accordingly. The equivalent widths of the $\mathrm{O}$ viI K- $\alpha$ lines indicate that many lines are saturated but not damped. The spectral resolution of the Reflection Grating Spectrometer (RGS) of XMM-Newton is not good enough to resolve the $\mathrm{O}$ vir line and obtain the velocity width. Therefore, the Voigt profile fitting, as has been done in Fang et al. (2015), might not be an accurate way to obtain the column density of these lines. Instead, the equivalent widths of the $\mathrm{O}_{\text {VII }} \mathrm{K}-\alpha$ and $\mathrm{O}_{\text {VII }} \mathrm{K}-\beta$ lines can be combined to constrain the column density and the velocity width (e.g., Nicastro et al. 2002; Williams et al. 2005; Gupta et al. 2012; Nicastro et al. 2016; Gupta et al. 2017); this is our approach for calculating the O viI column densities in this paper.

We provide an empirical estimation of the DM contribution of the Galactic disk and halo from the X-ray absorption analyses. For completeness, we have considered other phases, although those are not the primary contributors. Instead of constructing a density model, we provide the DM along the observed sightlines. It is a more appropriate representation of the Galactic DM than the previous estimates.

This paper is organized as follows. In section 2 we discuss the steps to calculate the Galactic dispersion measure. In section 3 we show how the Galactic dispersion measure contribution is distributed over the sky and compare it with previous models. Finally in section 4 we summarize the result.

\section{ANALYSIS}

The ISM and CGM are multiphase, so we accumulate the data from the literature in different wavelengths probing different phases. We do not assume any density model; we convert the observed quantities to the Galactic dispersion measure with the assumptions standard in the respective fields. In fact, by using more observables, we make fewer assumptions (\$2.2). We further note that the DM models based on the emission of the hot phase ignore all other phases, and the previous absorption based study includes some (but not all) of the cooler phases. As such, our study is more comprehensive and more accurate compared to previous studies.

The total Galactic dispersion measure (DM) contribution is be a combination of the disk and the halo in four different phases:

$$
D M_{G a l}=D M_{\text {cold }}+D M_{\text {cool }}+D M_{\text {warm }}+D M_{\text {hot }}
$$

Here, "cold" refers to $\approx 10^{4} \mathrm{~K}$ gas which is predominantly neutral, "cool" is $10^{4-5} \mathrm{~K}$ mildly ionized gas, "warm" is for $\approx 10^{5-5.5} \mathrm{~K}$ gas probed by Li-like ions (primarily $\mathrm{O} v \mathrm{vI}$ ), and hot refers to $\geqslant 10^{6} \mathrm{~K}$ gas probed by $\mathrm{H}-$ and $\mathrm{He}-$ like ions, e.g., $\mathrm{O}$ VII and $\mathrm{O}$ VIII (Tumlinson et al. 2017).

We obtain $\mathrm{DM}_{\text {cold }}$ from the 21-cm H I emission measurement at $z=0$ (HI4PI Collaboration et al. 2016) using the following equation:

$D M_{\text {cold }}=6.5 \mathrm{~cm}^{-3} p c\left(\frac{N_{H I}}{10^{21} \mathrm{~cm}^{-2}}\right)\left(\frac{x_{e}}{0.02}\right)$

Here, $x_{e}=\frac{n_{e}}{n_{H}}$ is the electron fraction, which is typically 0.02 for a $\approx 10^{4} \mathrm{~K}$ gas (Draine 2011).

The cool gas is probed by singly/doubly ionized gas (e.g., Si II and Si III ions). By assuming that the element is in only two ionization states, we obtain $\mathrm{DM}_{\text {cool }}$ from the column densities of Si II and $\mathrm{Si}$ III ions (also $\mathrm{C}_{\mathrm{II}}$ and $\mathrm{C}_{\mathrm{IV}}$ ions) using the following equation:

$$
\begin{array}{r}
D M_{\text {cool }}=5.3 \mathrm{~cm}^{-3} p c\left(\frac{N_{\text {SiII }}+N_{\text {SiIII }}}{2 \times 10^{13.4} \mathrm{~cm}^{-2}}\right) \\
\left(\frac{A_{S i, \odot}}{3.2 \times 10^{-5}}\right)^{-1}\left(\frac{Z}{0.1 Z_{\odot}}\right)^{-1}
\end{array}
$$

Here, we scale with respect to the median column density of Si II and $\mathrm{Si}$ III in the intermediate and high velocity absorbers in the Galactic halo (Richter et al. 2017), corrected by a factor of 2 to account for the low velocity absorbers (Zheng et al. 2015). $A_{S i, \odot}$ is the solar abundance of silicon (Asplund et al. 2009). The typical metallicity is taken to be $0.1 \mathrm{Z}_{\odot}$ (Wakker 2001). A similar calculation with $\mathrm{C}_{\text {II }}$ and

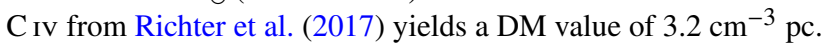

The assumption of all Si II and Si III coming from the same medium might not generally be true. The cool phase is photo-ionized, and the uncertainties related to photo-ionization are large. The column densities of $\mathrm{Si}$ II and $\mathrm{Si}$ III and their ratios span over an order of magnitude over the whole sky (Richter et al. 2017), indicating the complex thermal and ionization structure. For Carbon in the cool phase, observed absorption lines are from $\mathrm{C}_{\mathrm{II}}$ and $\mathrm{C}_{\mathrm{IV}}$, but not $\mathrm{C}_{\mathrm{III}}$, but in the photoionized gas $\mathrm{C}_{\text {III }}$ must exist together with $\mathrm{C}_{\text {II }}$ and C IV. This will make DM $\mathrm{DMol}_{\text {col }}$ based on Carbon, similar than from Si.

It should be noted that all of the $\mathrm{H}_{\mathrm{I}}$ measured in $21-\mathrm{cm}$ might not come from a predominantly neutral medium. If $\mathrm{H}$ I comes from an ionized medium, $\mathrm{DM}_{\text {cold }}$, the DM in the cold phase, would be lower. One would generally expect denser mediums (i.e., with $\left(\mathrm{N}\left(\mathrm{H}_{\mathrm{I}}\right)\right.$ $\geqslant 10^{20} \mathrm{~cm}^{-2}$ ) to be more shielded and hence predominantly neutral, while smaller $\mathrm{N}\left(\mathrm{H}_{\mathrm{I}}\right)$ values can come from a partially ionized medium. Based on the typical $\mathrm{N}\left(\mathrm{H}_{\mathrm{I}}\right)$ values in the cool phase, we estimate an approximate DM. The average ionization fraction of $\mathrm{H} \mathrm{I}$, $f_{H I}=0.3$ in the cool phase (Lehner \& Howk 2011; Putman et al. 2012; Richter et al. 2017). The median N(H I) in HI4PI Collaboration et al. (2016) is $\mathrm{N}\left(\mathrm{HI}_{\mathrm{I}}\right)=4.3 \times 10^{18} \mathrm{~cm}^{-2}$. The DM for this median $\mathrm{N}\left(\mathrm{HI}_{\mathrm{I}}\right)$ and average $f_{H I}$ would be $6.5 \mathrm{~cm}^{-3} \mathrm{pc}$, including the correction factor of 2 to account for the low velocity gas (Zheng et al. 2015). This is comparable with $\mathrm{DM}_{\text {cool }}$ obtained from silicon and carbon lines using equation 3, as was also found by Prochaska \& Zheng (2019). Therefore, between the cold and cool phases, the sightlines with high $\mathrm{N}\left(\mathrm{H}_{\mathrm{I}}\right)$ are likely to have a higher $\mathrm{DM}_{\text {cold }}$ and the smaller $\mathrm{N}\left(\mathrm{H}_{\mathrm{I}}\right)$ would contribute higher $\mathrm{DM}_{\text {cool }}$. As the DM calculated from metal lines and $\mathrm{H}_{\mathrm{I}}$ are comparable, we consider the DM calculated from metal lines as the bulk estimate of $\mathrm{DM}_{\text {cool }}$. To avoid double counting, we do not include the cool phase in the final calculation. 
If along a given sightline $\mathrm{DM}_{\text {cold }} \ll \mathrm{DM}_{\text {cool }}$ indicating that DM is dominated by the cool phase rather than the cold phase, $\mathrm{DM}_{\text {cool }}$ might be added to the final calculation of $\mathrm{DM}_{G a l}$.

We estimate the DM contribution of the warm phase using the following equation:

$$
\begin{aligned}
D M_{\text {warm }}=4.4 \mathrm{~cm}^{-3} p c( & \left.\frac{N_{O V I}}{2 \times 10^{14.3} \mathrm{~cm}^{-2}}\right)\left(\frac{f_{O V I}}{0.2}\right)^{-1} \\
& \left(\frac{A_{O, \odot}}{4.9 \times 10^{-4}}\right)^{-1}\left(\frac{Z}{0.3 Z_{\odot}}\right)^{-1}
\end{aligned}
$$

Here, we scale with respect to the median column density of $\mathrm{O} v \mathrm{vI}$ in the intermediate and high velocity absorbers in the Galactic halo (Sembach et al. 2003), corrected by a factor of 2 to account for the low velocity absorbers (Zheng et al. 2015). $A_{O, \odot}$ is the solar abundance of oxygen (Asplund et al. 2009). The typical metallicity is taken to be $0.3 \mathrm{Z}_{\odot}$, the median metallicity of the warm CGM of L* galaxies in the COS-Halos sample (Prochaska et al. 2017). We adopt the ionization fraction of $f_{\mathrm{OVI}}=0.2$, maximum for the collisional ionization equilibrium.

To calculate the DM contribution of the hot phase, we use $\mathrm{O}$ viI $\mathrm{K}$ $\alpha, \mathrm{O}$ VII K- $\beta$ and $\mathrm{O}$ viII Ly- $\alpha$ lines. Previous studies of DM assumed the ionization fraction of $\mathrm{O}$ VII, $f_{O V I I}=1$, and did not use the $\mathrm{O}$ VII $\mathrm{K}$ $\beta$ line to estimate $\mathrm{N}\left(\mathrm{O}_{\text {viI }}\right)$. Unlike them, we use both $\mathrm{O}_{\text {viI } \mathrm{K}-\alpha}$ and $\mathrm{K}-\beta$ lines to calculate $\mathrm{N}\left(\mathrm{O}_{\mathrm{VII}}\right)$, and the ratio of $\mathrm{N}\left(\mathrm{O}_{\mathrm{VIII}}\right)$ and $\mathrm{N}\left(\mathrm{O}\right.$ VII) to calculate $f_{O V I I}$ (see also Nicastro et al. 2002; Williams et al. 2005; Gupta et al. 2012). This is the main advancement in this paper compared to earlier DM estimates.

$\mathrm{DM}_{\text {hot }}$ is calculated by two methods. First, we calculate the DM from direct $\mathrm{O}$ VII (and $\mathrm{O}$ viII, if available) line measurement by Gupta et al. (2012); Nicastro et al. (2016); Gupta et al. (2017) using the following equation:

$$
\begin{array}{r}
D M_{\text {hot }}=83.7 \mathrm{~cm}^{-3} p c\left(\frac{N_{\text {OVII }}}{10^{16.5} \mathrm{~cm}^{-2}}\right)\left(\frac{f_{O V I I}}{1}\right)^{-1} \\
\left(\frac{A_{O, \odot}}{4.9 \times 10^{-4}}\right)^{-1}\left(\frac{Z}{0.3 Z_{\odot}}\right)^{-1}
\end{array}
$$

Because $\mathrm{O}$ vir $\mathrm{K}-\alpha$ line in the CGM is often saturated but not damped, the column density cannot be directly estimated from the equivalent width (EW) of O viI K- $\alpha$ by fitting it with a Gaussian/Voigt profile. For a given EW, the column density $\mathrm{N}$ in the flat portion of the curve-of-growth is a function of the velocity width $b\left(\mathrm{~N} \propto b \times e^{b^{-2}}\right.$, Draine 2011). The intersection of the $\mathrm{N}$ vs. $b$ contours for the EWs of $\mathrm{O}$ vir $\mathrm{K}-\alpha$ and $\mathrm{K}-\beta$ lines corresponds to the best-fitted column density and the velocity width of $\mathrm{O}$ vII. The uncertainty is obtained from the range allowed by the confidence intervals of both contours at the best-fitted value. O vin is optically thin, so N(O viII) is linearly proportional to $\mathrm{EW}(\mathrm{O} \mathrm{VIII})$.

If both $\mathrm{O}$ VII and $\mathrm{O}$ viII are detected or an upper limit exists along a sightline, the temperature (or its upper limit) of the gas is calculated from their column density ratio, $\frac{N_{O V I I I}}{N_{O V I I}}$, assuming that the gas is in collisional ionization equilibrium (CIE). The ionization fraction of O VII, $f_{O V I I}$ at that temperature is used to calculate $\mathrm{DM}_{\text {hot }}$. Along some sightlines, the measurement of the $\mathrm{O}$ vin line is not reported. This is either because $\mathrm{O}$ vin line was too weak to obtain a measurement of the column, or $\mathrm{O}$ virl could not be studied due to instrumental features at that wavelength. In these cases, we assume $f_{O V I I}=1^{1}$. As metallicity cannot be measured in X-ray absorption

\footnotetext{
1 The maximum ionization fraction of $\mathrm{O}$ VII in $\mathrm{CIE}$ is $\approx 0.9$. But the measurement uncertainty in the column density of $\mathrm{O}_{\text {VII }}$ is much larger than this uncertainty in the ionization fraction. This validates the assumption of $f_{\text {OVII }}=1$.
}

due to the lack of a hydrogen line, we adopt the same metallicity in the hot and warm phases, assuming that the warm phase forms by cooling from the hot phase.

The second method is based on the $\mathrm{N}(\mathrm{H})$ values of the hot phase estimated by Gatuzz \& Churazov (2018). There, the oxygen lines have been fitted using hybrid ionization modeling ${ }^{2}$, for a constant temperature of $10^{6.3} \mathrm{~K}$. We take $\mathrm{N}(\mathrm{H})$ in the hot phase for solar metallicity from Gatuzz \& Churazov $(2018)^{3}$, and convert it to $\mathrm{DM}_{\text {hot }}$ using

$D M_{\text {hot }}=85.6 \mathrm{~cm}^{-3} p c\left(\frac{N_{H}}{10^{20.3} \mathrm{~cm}^{-2}}\right)\left(\frac{Z}{0.3 Z_{\odot}}\right)^{-1}$

The $\mathrm{N}(\mathrm{H})$ of the cold phase was part of their model, whose value is not necessarily the same as the $21-\mathrm{cm}$ measurement by HI4PI Collaboration et al. (2016). Therefore, we calculate $\mathrm{DM}_{\text {cold }}$ of the Gatuzz \& Churazov (2018) sightlines from their estimated $\mathrm{N}(\mathrm{H})$ of the cold phase. Thus the total $\mathrm{DM}_{G a l}$ from Gatuzz \& Churazov (2018) is ionization model-based, while other estimations (Gupta et al. 2012; Nicastro et al. 2016) are empirical.

As can be seen from equations 2-6, The typical DM contribution of the hot phase $(>80)$ exceeds the typical DM contribution of any other phase $(<8)$ by almost an order of magnitude. Therefore, inclusion of those phases does not significantly affect the total Galactic DM contribution. Nonetheless, we add the contribution of the cold phase, because:

1) unlike the cool and warm phases, the $21-\mathrm{cm}$ data is available along all the sightlines where X-ray data are available,

2) the DM contribution from the cool and warm phases are complicated by the uncertainties related to photo-ionization. The calculation in the cold phase, however, is straightforward, and

3 ) the relative contribution of the cold and hot phase may vary wildly over the sky because of the known anisotropy of the hot phase in the Galactic halo (Henley et al. 2010; Gupta et al. 2012, 2017; Nakashima et al. 2018).

\section{RESULTS AND DISCUSSION}

We tabulate the Galactic DM from the hot and the cold phases in tables A1, A2 and A3. At smaller galactic latitudes, the cold phase has a significant contribution from the disk, and the contribution from the hot and the cold phases are comparable. At higher galactic latitudes $\left(b>20^{\circ}\right)$ the contribution from the cold phase is negligible compared to the hot phase.

We show the Galactic dispersion measure as a function of the galactic coordinates in Figure 1. We also plot the median DM contribution of the cool and warm phases to show that their values are negligible compared to the DM from the hot (and cold) phases (Figure 1, top). The pulsar dispersion measure toward LMC and SMC (Ridley et al. 2013) are comparable with the DM values we obtain, validating the assumption of the $0.3 \mathrm{Z}_{\odot}$ metallicity in the hot phase.

The DM profile from the density model of Yamasaki \& Totani (2020) is shown for comparison (Figure 1, bottom). The range of DM values at a given galactic latitude (longitude) is similar to the range of DM values over the entire range of galactic longitude (latitude). On average, the model does a pretty good job of predicting the Galactic DM. However, the exact DM value along many sightlines deviate significantly from the predicted profile, both at small and large $(1, b)$. This shows that not even all the disk dominated sightlines can be

\footnotetext{
2 The photo-ionization parameter is negligibly small in their model. Therefore, effectively, the model is collisional ionization.

3 we discard the sightlines with an upper limit of $\mathrm{N}(\mathrm{H})$
} 

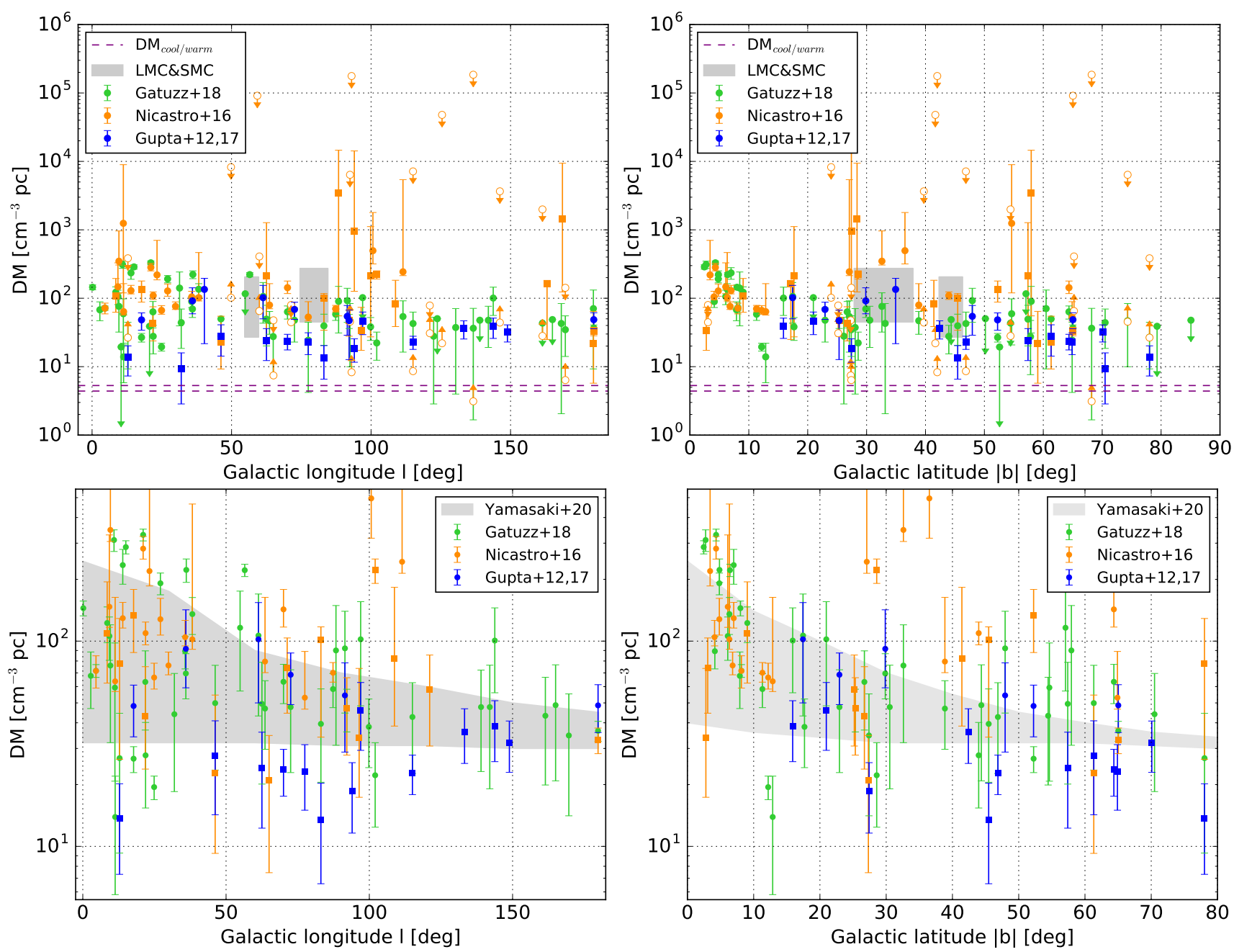

Figure 1. Dispersion measure (DM) contribution of the Galactic disk and halo as a function of galactic longitude (left) and latitude (right). The data points are derived from the 21-cm and X-ray absorption measurement of the cold (HI4PI Collaboration et al. 2016) and hot (Gupta et al. 2012; Nicastro et al. 2016; Gupta et al. 2017; Gatuzz \& Churazov 2018) Galactic halo. The filled circles denote the sightlines where both O viI and O viII lines were detected, the unfilled circles are where upper limits of $\mathrm{O}$ VIII were reported, squares denote the sightlines where the value of $\mathrm{O}$ vIII line was not reported and $f_{\mathrm{O}}$ vII is assumed to be 1 to calculate DM. Top: The gray regions denote the range of the pulsar dispersion measure towards LMC and SMC (Ridley et al. 2013, and references therein). The horizontal dashed line is the median of DM contribution of the cool and warm phases (Richter et al. 2017; Sembach et al. 2003). Bottom: The gray region is the predicted Galactic DM contribution modeled from the emission measure of hot Galactic halo (Yamasaki \& Totani 2020).

explained by the disk+spherical halo model. As the measurement of FRB DM is sightline specific, the estimation of Galactic DM and the cosmological calculations following that can be drastically incorrect if we use an average value. Therefore, the empirical values we present here are more accurate than the previously modeled values.

Most of the sightlines have been observed with multiple instruments (Chandra and XMM-Newton) and/or multiple methods (direct measurement of absorption lines or ionization modeling). The DM estimates along some of the sightlines are not consistent with each other within error. This might partially be due to the assumption about the temperature (Figure 2). For simplicity, Gatuzz \& Churazov (2018) assumed a constant temperature of $10^{6.3} \mathrm{~K}$ for all the sightlines. While this assumption is generally reasonable, the temperature obtained from the ratio of $\mathrm{N}\left(\mathrm{O}_{\mathrm{VIII}}\right)$ and $\mathrm{N}\left(\mathrm{O}_{\mathrm{VII}}\right)$ along every sightline is not necessarily the same. Because the ionization fraction of O VII changes sharply around $10^{6.3} \mathrm{~K}$ ( $f_{O V I I}$ drops from 0.85 at $10^{6.2} \mathrm{~K}$ to 0.3 at $10^{6.4} \mathrm{~K}$ ), the DM estimation is very sensitive to the temperature of the hot component. On the other hand, the ionization model might have a better continuum (and hence absorption line) estimation than the direct line measurements. The model simultaneously takes into account of multiple phases, including the absorption lines of multiple elements in addition to $\mathrm{O}$ viI and $\mathrm{O}$ vIII. Therefore, we do not have any particular reason to prefer one method over the other.

The $\mathrm{DM}_{G a l}$ does not have any trend with either of the galactic coordinates (Figure 1). There is more than two orders of magnitude scatter in the values of the $\mathrm{DM}_{G a l}$. This shows that the geometrically ordered structures like the spherical halo and the disk might not explain the observation well. As $\mathrm{DM}_{G a l}$ is dominated by $\mathrm{DM}_{h o t}$, this pattern of DM reflects the characteristics of the hot Galactic halo. This is consistent with the X-ray emission and absorption analyses which also report the hot Galactic halo to be inhomogeneous and anisotropic (Gupta et al. 2012; Henley \& Shelton 2013; Gupta et al. 2014, 2017; Nakashima et al. 2018).

In Figure 3 we show the sky distribution of Galactic DM contri- 


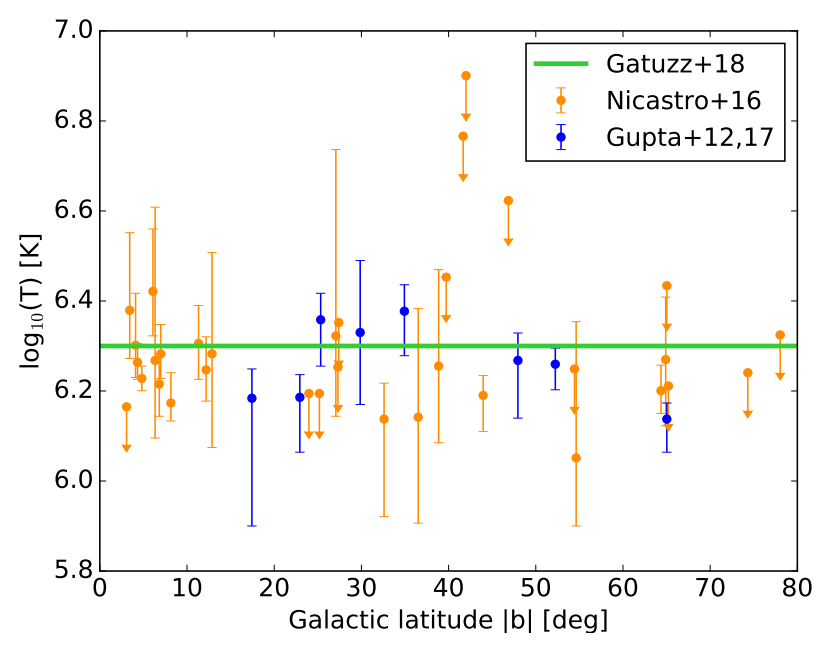

Figure 2. The temperature of the hot Galactic halo as a function of galactic latitude. The horizontal line corresponds to $\mathrm{T}=10^{6.3} \mathrm{~K}$ which was assumed to be constant for all sightlines in Gatuzz \& Churazov (2018). The data points are derived from the ratio or upper limit of the ratio of $\mathrm{N}\left(\mathrm{O}_{\mathrm{VIII}}\right)$ and $\mathrm{N}\left(\mathrm{O}_{\mathrm{VII}}\right)$ (Gupta et al. 2012; Nicastro et al. 2016) under the assumption of collisional ionization equilibrium.

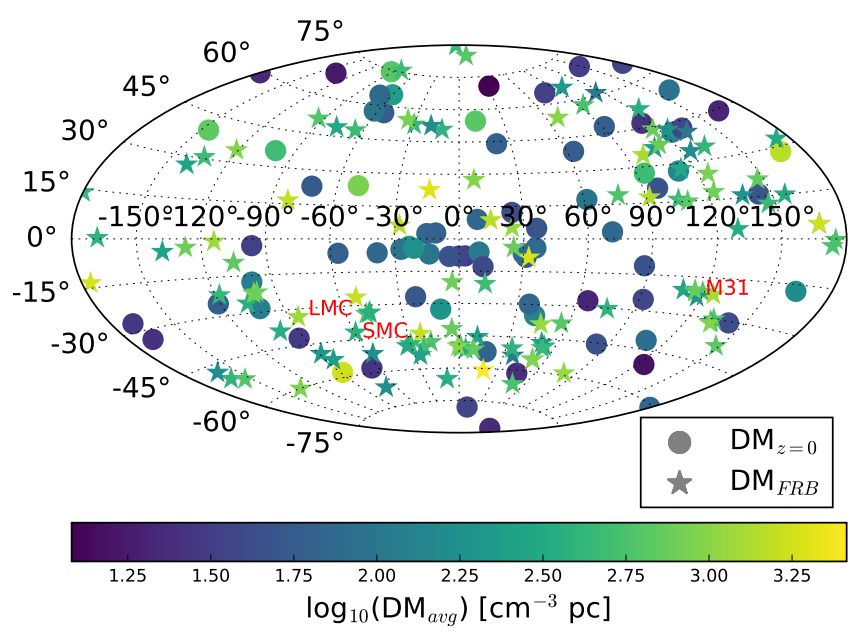

Figure 3. The average Galactic dispersion measure (DM) contribution obtained from multiple methods (direct measurement of $\mathrm{O}$ VII and $\mathrm{O}$ VIII lines vs. hybrid ionization modeling) and/or instruments (i.e., Chandra and XMMNewton) in the Aitoff projection of galactic coordinates. The DM of the extragalactic FRBs observed in the past decade are plotted for comparison. The symbols are color-coded with the $\log _{10}$ of DM; the filled circles are for the Galactic DM and the stars are for the FRB DM.

bution along with the DM of all FRBs discovered over past decade ${ }^{4}$. For the sightlines observed multiple times using different instruments and/or analyzed in different methods, we plot the average DM. We also calculate the maximum DM along each sightline (Table A4). The average DM should be the best estimate, while the maximum Galactic DM would provide a lower limit on the DM of the IGM.

As can be seen in Figure 1, the distribution of $\mathrm{DM}_{G a l}$ in the sky does not have any orderly pattern (Figure 3). Two sightlines far apart can have similar $\mathrm{DM}_{\text {Gal }}$, while close-by sightlines show a

\footnotetext{
4 The details of these FRBs are avaiable at http://www. frbcat.org/
}

Table 1. Correlation test. The number of sightlines in each segment are mentioned in parentheses

\begin{tabular}{|c|c|c|}
\hline Correlation with & $\tau$ & $p$ \\
\hline \multicolumn{3}{|c|}{ All sightlines (72) } \\
\hline$b$ & 0.059 & 0.460 \\
\hline$l$ & -0.044 & 0.586 \\
\hline \multicolumn{3}{|c|}{ Extra-galactic: $|b|>20^{\circ}$ (46) } \\
\hline$b$ & -0.005 & 0.962 \\
\hline$l$ & -0.080 & 0.432 \\
\hline \multicolumn{3}{|c|}{ Off-center: $20^{\circ}<l<340^{\circ}(59)$} \\
\hline$b$ & 0.026 & 0.768 \\
\hline$l$ & -0.070 & 0.436 \\
\hline \multicolumn{3}{|c|}{ Northern hemisphere: $b>0^{\circ}(35)$} \\
\hline$b$ & -0.150 & 0.206 \\
\hline$l$ & -0.106 & 0.371 \\
\hline \multicolumn{3}{|c|}{ Southern hemisphere: $b<0^{\circ}(37)$} \\
\hline$b$ & 0.162 & 0.158 \\
\hline$l$ & -0.042 & 0.714 \\
\hline
\end{tabular}

scatter in $\mathrm{DM}_{G a l}$. We do not find any systematic increase in $\mathrm{DM}_{G a l}$ toward the direction of $\mathrm{M} 31\left(l=121.17^{\circ}, b=-21.57^{\circ}\right)$, indicating that the halo of M 31 might not have a significant contribution to the measured $\mathrm{DM}_{G a l}$. Thus, interpolation of $\mathrm{DM}_{G a l}$ values might not be the correct approach due to its non-monotonic behavior as a function of the galactic coordinates. This shows how complex the distribution of density, spatial extent, temperature and ionization state of the Galactic disk and halo are, and how challenging the modeling is to explain the details of the multi-wavelength observations.

\subsection{Statistical considerations}

We perform a Kendall's $\tau$ test to verify any correlation between $\mathrm{DM}_{G a l}$ and the galactic coordinates (Table 1). We do not include the lower limits and upper limits in this test. The value of $\tau=+1 /-1 / 0$ implies a perfect positive/negative/null correlation, and the $\mathrm{p}$-value is the probability of a null correlation. The all-sky distribution of $\mathrm{DM}_{G a l}$ has $\mathrm{a}|\tau|<0.1$ and $\mathrm{a} \approx 50 \%$ probability of any correlation with $l$ or $b$. The lack of correlation between $\mathrm{DM}_{G a l}$ and $b$ becomes more prominent when the extragalactic sightlines $\left(|b|>20^{\circ}\right)$ or offcenter sightlines $\left(20^{\circ}<l<340^{\circ}\right)$ are considered; the probability of a null correlation enhances to $96 \%$ and $77 \%$, respectively. This shows why the disk model is not a good representation of the $\mathrm{DM}_{G a l}$ distribution. The lack of strong anti-correlation between $\mathrm{DM}_{G a l}$ and $l$ indicates that the halo is not isotropic, and hence, a spherical model might not be appropriate either. The correlations (or the lack there of) are not exactly similar in the two hemispheres. $\mathrm{DM}_{G a l}$ in the northern hemisphere shows a weak anti-correlation with $b$, while the southern hemisphere shows a weak positive correlation. The probability of a null correlation with $l$ is higher $(71 \%)$ in the southern hemisphere than the northern hemisphere (37\%). These asymmetries are difficult to account for in the geometric density models. Once again, the empirical estimates are better.

Our correlation coefficients (Table 1) are based on X-ray absorption studies along 72 sightlines. We compare these with the coefficients from Henley \& Shelton (2013) based on X-ray emission measures (EM) along 110 sightlines. The EM distribution did not show any dependence on $|b|$ in either hemispheres, but there was signif- 
icant $(p \ll 1)$ anti-correlation with $l$ in the southern hemisphere. This is different from our $D M_{G a l}$ distribution. As the emission is dominated by denser regions, the disparity of correlations between $\mathrm{EM}$ and $\mathrm{DM}_{G a l}$ distribution indicates that the hot gas probed in emission and absorption might not be the same. This also adds to the reasons for using absorption analyses for DM measurements.

Next, we calculate the mean and the median of the $\mathrm{DM}_{G a l}$ distribution. We do not include the lower limits and upper limits. The average $\mathrm{DM}_{G a l}$ ranges from 12 to $1749 \mathrm{~cm}^{-3}$ pc. This is larger than the ranges predicted by Prochaska \& Zheng (2019) based on the absorption analysis $\left(50-80 \mathrm{~cm}^{-3} \mathrm{pc}\right)$ and by Yamasaki \& Totani (2020) based on emission analysis $\left(30-245 \mathrm{~cm}^{-3} \mathrm{pc}\right)$. There are only 8 out of 72 sightlines with the average $\mathrm{DM}_{G a l}>245 \mathrm{~cm}^{-3} \mathrm{pc}$, and 9 sightlines with the average $\mathrm{DM}_{G a l}<30 \mathrm{~cm}^{-3}$ pc. That means the $\mathrm{DM}_{G a l}$ of most $(76 \%)$ of the sightlines are consistent with the estimate of Yamasaki \& Totani (2020). The histogram of $\mathrm{DM}_{G a l}$ is asymmetric toward the higher values (Figure 4), making the mean $\left(161 \mathrm{~cm}^{-3} \mathrm{pc}\right)$ significantly higher than the median $\left(64 \mathrm{~cm}^{-3} \mathrm{pc}\right)$.

Please note that the $\mathrm{DM}_{G a l}$ values are not as robust as the $\mathrm{N}\left(\mathrm{O}_{\mathrm{vII}}\right)$ values. $\mathrm{DM}_{G a l}$ depends on both $\mathrm{N}\left(\mathrm{O}_{\mathrm{VII}}\right)$ and $f_{\mathrm{O} \text { VII }}$ (see equation 5 ). The uncertainty in the value of $f_{\mathrm{O} \text { VII }}$ depends on the robustness of temperature as well as the value of $f_{\mathrm{O} \text { VII }}$ at the temperature of the hot gas. The temperature depends on $\mathrm{N}\left(\mathrm{O}_{\mathrm{VIII}}\right)$ and N(O VII $)$, the error in both oxygen lines are propagated in the uncertainty of the temperature, making it less constrained than the individual lines. If $f_{\mathrm{O} \text { VII }}$ changes rapidly within the range of the temperature of the hot gas, the uncertainty in $\mathrm{DM}_{G a l}$ will be driven by the uncertainty in

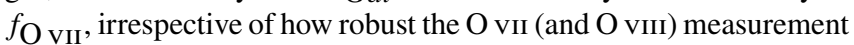
is. Secondly, the estimated $\mathrm{DM}_{G a l}$ from multiple studies can be different due to the difference in method and/or instrument. This adds another uncertainty in $\mathrm{DM}_{G a l}$ when the values are averaged.

Keeping the above discussion in mind, we consider the distributions of $\mathrm{DM}_{G a l}-\sigma_{l}$ and $\mathrm{DM}_{G a l}+\sigma_{u}$. Here $\sigma_{l}$ and $\sigma_{u}$ are the statistical uncertainty of the average $\mathrm{DM}_{G a l}$ in the lower and the upper end, respectively. The median of these two distributions provide an uncertainty in the median of the $\mathrm{DM}_{G a l}$ distribution. We find that median $D M_{G a l}=64_{-23}^{+20} \mathrm{~cm}^{-3}$ pc (Figure 4). Additionally, we calculate the uncertainty in the mean by propagating the uncertainty of individual sightlines assuming Poissonian statistics, and obtain mean $D M_{\text {Gal }}=161_{-32}^{+243} \mathrm{~cm}^{-3}$ pc. The $68 \%(90 \%)$ confidence interval of the $\mathrm{DM}_{\text {Gal }}$ distribution is 33-172 (23-660) $\mathrm{cm}^{-3}$ pc. Our typical $\mathrm{DM}_{G a l}$ is larger than the mean based on density models and cosmological hydrodynamic simulations (43 and $30 \mathrm{~cm}^{-3} \mathrm{pc}$, respectively; Dolag et al. 2015; Yamasaki \& Totani 2020).

\subsection{Utility}

For an FRB localized in the sky, one needs to find the closest sightlines (i.e., the sightlines at smallest angular separation from the FRB) and obtain the mean of the Galactic DM along those sightlines. This would be the Galactic contribution to the total DM toward that FRB. The choice of the statistic (e.g., mean, median or interpolation) to combine multiple sightlines and the angular separation within which to consider the sightlines may vary with the scientific purpose. Using the $\mathrm{DM}_{G a l}$ of a single sightline would be the simplest option, although that might not be the most accurate estimate.

We attach a machine readable file with the paper. It has the Galactic longitude and latitude, average $\mathrm{DM}_{G a l}$ and associated statistical and systematic uncertainty, and the maximum $\mathrm{DM}_{G a l}$ along all sightlines considered here. The systematic uncertainty along a sightline reflects the scatter between the individual estimates along that sight-

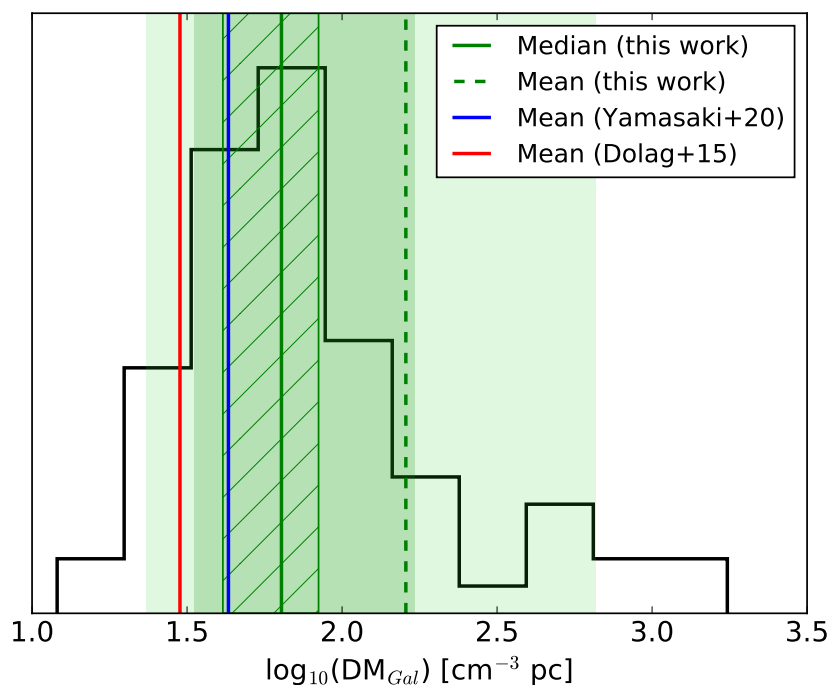

Figure 4. The histogram of Galactic dispersion measure in $\log _{10}$ scale. The distribution is asymmetric, with a tail toward higher values. This is reflected by the stark difference between the median and the mean of the distribution. The hatched region corresponds to the uncertainty in the median. The dark (light) shaded region corresponds to $68 \%(90 \%)$ confidence interval. Overall, our estimate of Galactic DM is larger than the previous estimates. The mean of those estimates are shown for comparison.

line. Thus, for the sightlines measured once, there is no systematic uncertainty. Statistical uncertainty along a sightline is obtained by propagating the uncertainty in individual measurement along that sightline in quadrature.

We build a code to extract the $\mathrm{DM}_{\text {Gal }}$ toward an FRB. We attach a copy of the code with the paper. It takes the galactic coordinate of the FRB of consideration as system arguments in the units of degree, reads the galactic coordinates of the 72 sightlines from the file mentioned in the previous paragraph, calculates the angular distance between the FRB and all the sightlines and returns the best estimate of Galactic DM and associated error using the following 3 methods. I) The $\mathrm{DM}_{G a l}$ and its statistical uncertainty along the sightline at smallest angular separation from the FRB.

II) The mean and the median of the sightlines within a threshold of angular separation from the FRB.

III) The mean and the median of the sightlines separated from the closest sightline within a tolerance limit.

The threshold and the tolerance of angular separation in method II and III are taken as system arguments in the units of degree. We recommend the user to try different values of threshold and tolerance instead of fixed preconceived values, and wisely choose the statistic and the method depending on the scientific interest. This code can easily be combined with ISM dominated models (e.g., Cordes \& Lazio 2002). It can also be extended to compare with the DM values from previous studies (Prochaska \& Zheng 2019; Yamasaki \& Totani 2020). If a nearby sightline within the user's choice of threshold is not found, our code prompts a message asking to use the median of our $\mathrm{DM}_{G a l}$ distribution as an alternative to the earlier estimates from density models.

It should be noted that while the previous density models are allsky by construction, they are constrained by observations of similar sky coverage as ours. That means, the models and our empirical table have similar amount of observational information. The $\mathrm{DM}_{G a l}$ values toward the directions without any observational information 
are interpolated in the models assuming some geometric symmetry. Therefore, if there is no sightline in our database within the angular separation threshold of the user, we would recommend the following paths:

1) Calculate the $\mathrm{DM}_{\text {Gal }}$ from the density models toward the FRB, and also along the closest sightline to the FRB, in our database (the output of method I in the previous paragraph). Then, scale it with our estimate toward the closest sightline, i.e., DM (our estimate toward the FRB) =

DM (model estimate toward the FRB) $\overline{\mathrm{DM} \text { (model estimate toward the closest sightline) }} \times \mathrm{DM}$ (our estimate toward the closest sightline)

2) Modify the angular separation threshold and re-calculate $\mathrm{DM}_{G a l}$ from our table (the output of method II in the previous paragraph). Calculate the $\mathrm{DM}_{G a l}$ from the density models along the sightlines within the new threshold, and use the same statistic of median/mean as for our empirical estimate. Then, use the similar scaling as shown in the previous point, by replacing the closest sightline with the sightlines within the threshold. We have tested this for a few FRBs and found that at $|b| \geqslant 20^{\circ}$, where the halo dominates over the ISM, setting a threshold of upto 20-25deg produces a meaningful result.

Please note that the DM from cool and warm phases are not included in this code (see $\$ 2$ for details). If needed, one can add the median DM from these phases to the output of the code, although the resulting correction will be minimal.

As an example, we discuss the case of FRB 20200125A at $(l, b)$ $=(359.8,58.4)$ (Parent et al. 2020). It has a DM of $179 \mathrm{~cm}^{-3} \mathrm{pc}$. For the ISM contribution to the DM being $\approx 25 \mathrm{~cm}^{-3} \mathrm{pc}$ (Cordes \& Lazio 2002), and by assuming no contribution from the Galactic halo, the maximum redshift of the host is $z_{\max }=0.17$. Assuming a fiducial Galactic halo contribution of $50 \mathrm{~cm}^{-3} \mathrm{pc}$, it reduces to $z_{\max }$ $=0.12$. The nearest sightline in our database, which is 7.3deg away from the direction toward the FRB has $\mathrm{DM}_{G a l}=645_{-459}^{+3148} \mathrm{~cm}^{-3} \mathrm{pc}$. Because this is not constraining enough for this particular FRB, we consider the median of all sightlines within 20deg of the FRB, and find $\mathrm{DM}_{\text {Gal }}=71 \pm 7 \mathrm{~cm}^{-3} \mathrm{pc}$, including all phases. Similar exercise for the density models of Prochaska et al. (2017) and Yamasaki \& Totani (2020) predicts 39 and $41 \mathrm{~cm}^{-3} \mathrm{pc}$, respectively. These are smaller than our prediction by $4 \sigma$. The predicted redshifts of the FRB based on the density models are larger than that based on our empirical estimate by $37 \%$. Once the redshift of the host of this FRB is determined, the density models and our empirical results can be tested to see which one is a better description of the Galactic halo.

\section{CONCLUSIONS}

Based on 21-cm, UV and X-ray absorption analyses at $z=0$ along 72 sightlines, we provide an empirical list of the Galactic dispersion measure contribution to the extragalactic fast radio bursts. It is independent of any density model and the spatial extent of the Galactic halo. We improve upon the measurements of $\mathrm{N}(\mathrm{O}$ viI $)$ and $\mathrm{f}_{O V I I}$ compared to previous studies, thus providing a better estimate of DM in the hot phase. Our findings are:

1) $D M_{G a l}$ is dominated by the hot phase probed by X-ray absorption.

2) There is no definite trend of $D M_{G a l}$ with respect to the galactic coordinates.

3) The previous models on average are consistent with our measurements, but there is significant scatter around the average. There are also a few sightlines where our measurements are significantly different.

4) The median $\mathrm{DM}_{G a l}=64_{-23}^{+20} \mathrm{~cm}^{-3} \mathrm{pc}$. The $68 \%(90 \%)$ confidence interval is $33-172(23-660) \mathrm{cm}^{-3} \mathrm{pc}$.

Different methods of DM estimates have their own strengths and weaknesses. The disk-plus-halo models based on X-ray emission make a lot of assumptions, but the models can provide a rough estimate of the DM along any sky direction. Absorption measurements have significantly smaller sky coverage, but they have significantly fewer assumptions compared to emission based studies; this way they are more accurate. The work we present here is complementary to previous works, and provides more accurate DM values along the sightlines probed. We provide a table and a code to retrieve $D M_{G a l}$ for any FRB localized in the sky.

\section{ACKNOWLEDGEMENTS}

SM and SD gratefully acknowledges the support from the NASA grant NNX16AF49G. YK acknowledges support from grant DGAPA-PAPIIT 106518, and from program DGAPA-PASPA.

\section{DATA AVAILABILITY}

The data behind figures are tabulated in A1,A2,A3 and A4.

\section{REFERENCES}

Asplund M., Grevesse N., Sauval A. J., Scott P., 2009, ARA\&A, 47, 481 Boylan-Kolchin M., Bullock J. S., Sohn S. T., Besla G., van der Marel R. P., 2013, ApJ, 768, 140

Cordes J. M., Lazio T. J. W., 2002, arXiv e-prints, pp astro-ph/0207156

Dolag K., Gaensler B. M., Beck A. M., Beck M. C., 2015, MNRAS, 451, 4277

Draine B. T., 2011, Physics of the Interstellar and Intergalactic Medium

Fang T., Buote D., Bullock J., Ma R., 2015, ApJS, 217, 21

Gatuzz E., Churazov E., 2018, MNRAS, 474, 696

Gupta A., Mathur S., Krongold Y., Nicastro F., Galeazzi M., 2012, ApJ, 756, L8

Gupta A., Mathur S., Galeazzi M., Krongold Y., 2014, Ap\&SS, 352, 775

Gupta A., Mathur S., Krongold Y., 2017, ApJ, 836, 243

HI4PI Collaboration et al., 2016, A\&A, 594, A116

Henley D. B., Shelton R. L., 2013, ApJ, 773, 92

Henley D. B., Shelton R. L., Kwak K., Joung M. R., Mac Low M.-M., 2010, ApJ, 723, 935

Keating L. C., Pen U.-L., 2020, MNRAS, 496, L106

Lehner N., Howk J. C., 2011, Science, 334, 955

Li Z., Gao H., Wei J.-J., Yang Y.-P., Zhang B., Zhu Z.-H., 2019, ApJ, 876, 146

Lorimer D. R., Bailes M., McLaughlin M. A., Narkevic D. J., Crawford F., 2007, Science, 318, 777

Macquart J. P., et al., 2020, Nature, 581, 391

Masui K. W., Sigurdson K., 2015, Phys. Rev. Lett., 115, 121301

Nakashima S., Inoue Y., Yamasaki N., Sofue Y., Kataoka J., Sakai K., 2018, ApJ, 862, 34

Nicastro F., et al., 2002, ApJ, 573, 157

Nicastro F., Senatore F., Gupta A., Guainazzi M., Mathur S., Krongold Y., Elvis M., Piro L., 2016, MNRAS, 457, 676

Parent E., et al., 2020, arXiv e-prints, p. arXiv:2008.04217

Petroff E., et al., 2016, Publ. Astron. Soc. Australia, 33, e045

Petroff E., Hessels J. W. T., Lorimer D. R., 2019, A\&ARv, 27, 4

Prochaska J. X., Zheng Y., 2019, MNRAS, 485, 648

Prochaska J. X., et al., 2017, ApJ, 837, 169

Putman M. E., Peek J. E. G., Joung M. R., 2012, ARA\&A, 50, 491

Richter P., et al., 2017, A\&A, 607, A48 
Table A1. $\mathrm{DM}_{\text {hot }}$ based on the O VII and O VIII measurements of Gupta et al. $(2012,2017)$ and $\mathrm{DM}_{\text {cold }}$ based on the $21-\mathrm{cm}$ measurements of HI4PI Collaboration et al. (2016). The last two columns denote the uncertainty in $\mathrm{DM}_{\text {hot }}$. (Full table is available online)

\begin{tabular}{|c|c|c|c|c|c|c|}
\hline Target & $\begin{array}{c}1 \\
\text { [deg] }\end{array}$ & $\begin{array}{c}\mathrm{b} \\
{[\mathrm{deg}]}\end{array}$ & $\begin{array}{l}\text { DM_cold } \\
{\left[\mathrm{cm}^{-3} \mathrm{pc}\right]}\end{array}$ & $\begin{array}{l}\text { DM_hot } \\
{\left[\mathrm{cm}^{-3} \mathrm{pc}\right]}\end{array}$ & $\begin{array}{c}\text { e_low } \\
{\left[\mathrm{cm}^{-3} \mathrm{pc}\right]}\end{array}$ & $\underset{\left[\mathrm{cm}^{-3} \mathrm{pc}\right]}{\text { e_up }}$ \\
\hline \multicolumn{7}{|c|}{ Both $\mathrm{O}_{\text {VII }}$ and $\mathrm{O}_{\text {VIII }}$ available: } \\
\hline 3C382 & 61.30 & 17.44 & 4.01 & 98.01 & 52.02 & 52.07 \\
\hline ARK564 & 92.13 & -25.33 & 3.23 & 43.86 & 34.40 & 20.12 \\
\hline Mrk $509^{a}$ & 35.97 & -29.86 & 2.56 & 156.61 & 48.98 & 48.25 \\
\hline \multicolumn{7}{|c|}{ Only $\mathrm{O}_{\mathrm{VII}}$ available. $f_{O V I I}=1$ : } \\
\hline $1 \mathrm{ES} 1927+654$ & 96.98 & 20.96 & 4.16 & 41.95 & 16.75 & 16.75 \\
\hline $3 \mathrm{C} 273$ & 289.95 & 64.36 & 1.09 & 22.53 & 6.07 & 6.07 \\
\hline
\end{tabular}

Table A2. $\mathrm{DM}_{\text {hot }}$ based on the $\mathrm{O}_{\text {VII }}$ and O VIII measurements of Nicastro et al. (2016) and $\mathrm{DM}_{\text {cold }}$ based on the 21-cm measurements of HI4PI Collaboration et al. (2016). The last two columns denote the uncertainty in $\mathrm{DM}_{\text {hot }}$. (Full table is available online)

\begin{tabular}{|c|c|c|c|c|c|c|}
\hline Target & $\begin{array}{c}1 \\
{[\mathrm{deg}]}\end{array}$ & $\begin{array}{c}\mathrm{b} \\
{[\mathrm{deg}]}\end{array}$ & $\begin{array}{l}\text { DM_cold } \\
{\left[\mathrm{cm}^{-3} \mathrm{pc}\right]}\end{array}$ & $\begin{array}{l}\text { DM_hot } \\
{\left[\mathrm{cm}^{-3} \mathrm{pc}\right]}\end{array}$ & $\begin{array}{c}\text { e_low } \\
{\left[\mathrm{cm}^{-3} \mathrm{pc}\right]}\end{array}$ & $\underset{\left[\mathrm{cm}^{-3} \mathrm{pc}\right]}{\text { e_up }}$ \\
\hline \multicolumn{7}{|c|}{ Both $\mathrm{O}_{\text {VII }}$ and $\mathrm{O}_{\text {VIII }}$ vailable: } \\
\hline SAXJ1808.4-3658 & 355.39 & -8.15 & 7.78 & 63.79 & 12.48 & 13.23 \\
\hline XTEJ1650-500 & 336.72 & -3.43 & 39.56 & 179.79 & 33.71 & 481.70 \\
\hline \multicolumn{7}{|c|}{ Only $O_{V I I}$ available. $f_{O V I I}=1$ : } \\
\hline PSRB0833-45 & 263.55 & -2.79 & 1.95 & 31.76 & 16.35 & 39.89 \\
\hline \multicolumn{7}{|c|}{$\mathrm{O}_{\text {VII }}$ and $3 \sigma$ upper limit of $\mathrm{O}_{\text {VIII }}$ available: } \\
\hline \multirow[t]{2}{*}{$3 \mathrm{C} 120$} & 190.37 & -27.40 & 6.49 & $<113.02$ & & \\
\hline & \multicolumn{6}{|c|}{$f_{O V I I}=1$, neglecting the upper limit of $\mathrm{O}_{\mathrm{VIII}}$} \\
\hline MAXIJ0556-332 & 238.94 & -25.18 & 2.59 & 46.32 & 22.78 & 35.75 \\
\hline
\end{tabular}

Table A3. $\mathrm{DM}_{\text {cold }}$ and $\mathrm{DM}_{\text {hot }}$ based on the ionization modeling of Gatuzz \& Churazov (2018) (Full table is available online)

\begin{tabular}{ccccccc}
\hline Target & $\begin{array}{c}\mathrm{l} \\
{[\mathrm{deg}]}\end{array}$ & $\begin{array}{c}\mathrm{b} \\
{[\mathrm{deg}]}\end{array}$ & $\begin{array}{c}\text { DM_cold } \\
{\left[\mathrm{cm}^{-3} \mathrm{pc}\right]}\end{array}$ & $\begin{array}{c}\text { err } \\
{\left[\mathrm{cm}^{-3} \mathrm{pc}\right]}\end{array}$ & $\begin{array}{c}\text { DM_hot } \\
{\left[\mathrm{cm}^{-3} \mathrm{pc}\right]}\end{array}$ & $\begin{array}{c}\text { err } \\
{\left[\mathrm{cm}^{-3} \mathrm{pc}\right]}\end{array}$ \\
\hline 4U 1254-69 & 303.48 & -6.42 & 20.75 & 0.06 & 39.96 & 6.32 \\
4U 1543-62 & 321.76 & -6.34 & 15.37 & 0.45 & 21.13 & 11.97 \\
4U 1636-53 & 332.91 & -4.82 & 24.97 & 0.65 & 32.45 & 13.19 \\
4U 1735-44 & 346.05 & -6.99 & 23.67 & 0.71 & 35.45 & 13.70 \\
4U 1820-30 & 2.79 & -7.91 & 6.49 & 0.39 & 24.28 & 5.31 \\
\hline
\end{tabular}

Ridley J. P., Crawford F., Lorimer D. R., Bailey S. R., Madden J. H., Anella

R., Chennamangalam J., 2013, MNRAS, 433, 138

Sembach K. R., et al., 2003, ApJS, 146, 165

Tumlinson J., Peeples M. S., Werk J. K., 2017, ARA\&A, 55, 389

Wakker B. P., 2001, ApJS, 136, 463

Williams R. J., et al., 2005, ApJ, 631, 856

Yamasaki S., Totani T., 2020, ApJ, 888, 105

Yao J. M., Manchester R. N., Wang N., 2017, ApJ, 835, 29

Yu H., Wang F. Y., 2017, A\&A, 606, A3

Zheng Z., Ofek E. O., Kulkarni S. R., Neill J. D., Juric M., 2014, ApJ, 797, 71

Zheng Y., Putman M. E., Peek J. E. G., Joung M. R., 2015, ApJ, 807, 103
Table A4. Average and maximum Galactic dispersion measure, combining multiple methods and instruments.(Full table is available online)

\begin{tabular}{cccccccc}
\hline Target & $\begin{array}{c}1 \\
{[\mathrm{deg}]}\end{array}$ & $\begin{array}{c}\mathrm{b} \\
{[\mathrm{deg}]}\end{array}$ & $\begin{array}{c}\mathrm{DM}_{\text {avg }} \\
{\left[\mathrm{cm}^{-3} \mathrm{pc}\right]}\end{array}$ & $\begin{array}{c}\text { e_low } \\
{\left[\mathrm{cm}^{-3} \mathrm{pc}\right]}\end{array}$ & $\begin{array}{c}\text { e_up } \\
{\left[\mathrm{cm}^{-3} \mathrm{pc}\right]}\end{array}$ & $\begin{array}{c}\mathrm{DM}_{\max } \\
{\left[\mathrm{cm}^{-3} \mathrm{pc}\right]}\end{array}$ & $\begin{array}{c}\text { e_sys } \\
{\left[\mathrm{cm}^{-3} \mathrm{pc}\right]}\end{array}$ \\
\hline 4U 1254-69 & 303.48 & -6.42 & 60.71 & 6.32 & 6.32 & 67.04 & - \\
4U 2129+12 & 65.01 & -27.31 & 21.07 & 13.63 & 31.93 & 53.00 & - \\
& \multicolumn{7}{c}{ combined } \\
4U 1543-62 & 321.76 & -6.34 & 69.26 & 6.59 & 148.45 & 465.46 & 32.77 \\
4U 1636-53 & 332.91 & -4.82 & 92.63 & 10.08 & 14.78 & 161.55 & 35.22 \\
4U 1735-44 & 346.05 & -6.99 & 94.22 & 7.87 & 11.65 & 154.34 & 35.10 \\
\hline
\end{tabular}

